\title{
A study on acute disseminated encephalomyelitis in children
}

\author{
Jena P.K. ${ }^{1}$, Murmu M.C. ${ }^{2}$, Priyadarshini $D^{3}$ \\ ${ }^{1}$ Dr. Pradeep Kumar Jena, Associate Professor, ${ }^{2}$ Dr. Mangal Charan Murmu, Associate Professor, ${ }^{3}$ Dr. Debashree \\ Priyadarshini, Resident Doctor; all authors are affiliated with Department of Paediatrics, S.C.B. Medical College, \\ Cuttack, Odisha, India.
}

Corresponding Author: Dr. Mangal Charan Murmu, Associate Professor, Department of Paediatrics, S.C.B. Medical College, Cuttack, Odisha, India. E-mail: mangal74murmu@yahoo.co.in

\begin{abstract}
Introduction: Acute disseminated encephalomyelitis (ADEM) is an acute demyelinating disorder of central nervous system characterized by scattered focal / multifocal inflammation of brain \& spinal cord that usually follows an apparently benign infection in otherwise healthy children \& young adults. It represents $30 \%$ of all childhood encephalitic illnesses. Material \& Method: The study was conducted over a period of two years from October 2016 to September 2018 at S C B Medical College, Cuttack. The patient fulfilling the inclusion criteria were taken into study. Result: Early institution of with immunosuppressive drugs hastens recovery and reduces morbidity. Despite the serious neuropsychiatric manifestations ADEM in children generally has good outcome. Children with ADEM need long term follow up for cognitive impairments and emotional problems. Conclusion: ADEM most commonly presently as an acute polysymptomatic encephalopathy and initially diagnosis may not be clear. Clinical evaluation, MRI \& CSF study are most useful to establish the diagnosis and rule out important differential diagnosis. Early institution of therapy with immunosuppressive therapy hasten recovery and reduces mortality.
\end{abstract}

Keywords: Encephalomyelitis, Demyelinating Diseases, Magnetic Resonance Imaging, corticosteroids.

\section{Background}

Acute disseminated encephalomyelitis (ADEM) is an acute demyelinating disorder of central nervous system characterized by scattered focal / multifocal inflammation of brain \& spinal cord that usually follows an apparently benign infection in otherwise healthy children \& young adults. It represents $30 \%$ of all childhood encephalitic illnesses [1]. It is considered as an autoimmune disorder that is triggered by environment stimulus in genetically susceptible individuals.

It is usually presenting as a monophasic disorder associated with multifocal neurological symptoms \& encephalopathy. In the past, ADEM commonly followed common childhood infection like measles, chickenpox \& smallpox. Because of significant advances in infectious disease control \& extensive immunisation coverage, nonspecific upper respiratory illness are the most common triggering event in developing countries, but the exact aetiological agent

Manuscript received: $30^{\text {th }}$ April 2019

Reviewed: $9^{\text {th }}$ May 2019

Author Corrected: $16^{\text {th }}$ May 2019

Accepted for Publication: $20^{\text {th }}$ May 2019 still remain unknown. But in developing countries, the high frequency of vaccination \& exanthematous fever account for frequent occurrences of post infectious demyelinating diseases [2].

In the past in the development countries, the incidence rate of post infectious demyelinating disease was 1 in 10,000 out of which 1 out of 1000 followed measles infection. Post vaccination encephalomyelitis incidence rate was 1 in 25000 especially following duck embryo anti rabies vaccination \& following live measles vaccination was 1 to 2 per one million ${ }^{3}$. The incidence of ADEM is estimated to be 0.4 per 100,000 per year [1].

However, very studies depict the exact details of ADEM especially the diagnostic criteria, course of the disease \& final outcome.

This study intends to analyse the epidemic variable, risk factors, clinical course, laboratory, radiological finding $\&$ treatment, in order to improve the diagnostic \& treatment \& to distinguish ADEM from other aetiologies of encephalopathy. 


\section{Aim of the study}

This present study has been undertaken with the following aims and

\section{Objectives}

1. To know the incidence of ADEM among hospitalized children. 2.To study the epidemiological factors associated. 3.To correlate the mode and type of presentation with outcome. 4.To analyze the derangement of various laboratory parameters. 5.To review the neuro imaging findings in detail and correlate with clinical picture $\&$ prognosis.

6.To throw light on various treatment modalities and study their efficacy. 7.Finally to assess in-hospital morbidity and mortality \& the clinical and imaging predictors of in hospital outcome in a group of childrenwith the diagnosis of ADEM.

\section{Material \& Method}

This is a prospective hospital based clinical study conducted in the department of paediatrics at SVVPGIP \& SCBMCH, Cuttack. It is one of the tertiary care health centres in eastern Odisha, catering to paediatric population from multiple districts of Odisha and additional districts of neighbouring states of West Bengal, Bihar \& Jharkhand.

The study was performed in close association with the department of Radio diagnosis, Cardiology, Neurology, Pathology, Microbiology, Haematology and Biochemistry over a period of two years from October 2016 to September 2018. Permission was taken from the institutional ethical committee to conduct this study.

Inclusion Criteria: Study group consisted of hospitalised children $<15$ years of age admitted to the indoors of SCB MCH \& SVPPGIP, Cuttack with the diagnosis of ADEM as per the defined criteria i.e.,1 Acute / sub-acute onset of polysymptomatic ${ }^{2}$ neurological presentation with prominence of cortical signs (changes in mental status, seizures, acute behavioural changes etc) preferably with a preceding history of infectious illness or vaccination [1,2,3 $4,5,6,7]$. 2. Monophasic time course of illness. 3. Signs \& symptoms cannot be explained by other known aetiologies. 4. MRI evidence of ADEM (bilateral asymmetric, multifocal, hyper intenselesions on FLAIR or T2 weighted images predominantly involving white matter with or without involvement of grey matter, thalamus \& basal ganglia without previous white matter changes) was considered corroborative $[3,4,5]$.
Exclusion Criteria:1. Recurrence of neurological signs \& symptoms beyond 3 months ofInitial illness. 2. Acute onset of flaccid paralysis of limbs or isolated optic neuritis orisolated transverse myelitis. 3. Presence of a significant preceding neurological abnormality or features suggestive of neurodegenerative disorder.4. Signs \& symptoms attributable to any systemic involvement.

All these patients selected were first stabilised. Detailed history and clinical examination special reference to central nervous system was done.

The level of consciousness was assessed using Glassgow coma scale. Motor or sensory deficits were classified as partial or complete.

The presence of aphasia, hemi paresis and visual defect was evaluated whenever possible according to child's age. All associated symptoms like seizure, headache, fever, altered level of consciousness were recorded.

Relevant investigations were performed to exclude infective or inflammatory aetiologies which included complete blood count and measurement of serum electrolytes, erythrocyte sedimentation rate and Cerebrospinal fluid analysis (CSF).

All patients had serological testing for mycoplasma and various implicated virus as well as nasopharyngeal \& rectal culture. ELISA (enzyme-linked immunosorbent assay), real timePolymerase chain reaction (PCR), conventional PCR were done to isolate the organism.

A viral pathogen was regarded as etiologic if one of the following criteria was met: 1.CSF \&/or serum contained virus specific IgM by ELISA.2. Raising IgG specific antibody levels or relatively high single IgG specific antibody level. 3. Positive PCR result. Routine Lumbar puncture was done, taking into consideration cardiorespiratory stability and after examining the fundus.

CSF analysis was done in term of cytological, biochemical, culture \& sensitivity, ADA (adenosine deaminase) assay and PCR study for isolating implicated viruses.

Neuroimaging was done in all patient after initial stabilisation. MRI (Magnetic Resonance Imaging) was the imaging modality of choice. A $1.5-\mathrm{T}$ seimens machine was used for the brain MRI study.T1, T2, fluid attenuated inversion recovery (FLAIR) and diffusion weighted images were obtained in the axial, sagittal and coronal plane. When feasible, contrast enhanced images 
were obtained using gadopentetate dimeglumine $(0.1 \mathrm{mmol} / \mathrm{kg})$. CT (Computed Tomography) was done in third generation scanner and contrast enhanced images were obtained in the selected few cases. Scans were reviewed by neurologist who was blinded to the clinical findings.

The images were assessed for lesion site, size, number, distribution, symmetry, any midline shift, haemorrhage and pattern of contrast enhancement. Standard 30minute interictal surface electroencephalo-phalogram (EEG) was recorded in patients with impaired consciousness or seizures.

These differential levels of investigations are the standard protocol in the diagnostic work up of patient with a neurological catastrophe and do not influence the etiologic diagnosis.

The incidence was defined as number of new cases of ADEM admitted to the hospital which came into existence within certain period of time per 100 patients admitted to the hospital.

All those cases then segregated as per age and gender. The mean age at onset is calculated and sex predilection determined.

The incidence of prodermal period and history of recent vaccination was assessed. After neuroimaging study, the incidence of abnormal findings on MRI/CT was calculated.

Mode of onset mode of presentation i.e. the type of deficit, cranial nerve involvement, and level of consciousness, seizures, headache, and all the features then correlated with the immediate outcome. Thorough history and a formal neurological examination to evaluate the outcome in terms of full recovery, motor deficit, cognitive defect, visual field defect, recurrent seizure, learning disorder, personality changes, psychiatric manifestations, death etc.

Repeat brain imaging was arranged if there was suggestion of progression or recurrence of neurological deficits. After ADEM was diagnosed, all cases were treated with high dose intravenous corticosteroids, either methyl prednisolone $(10-30 \mathrm{mg} / \mathrm{kg})$ or dexamethasone $(1 \mathrm{mg} / \mathrm{kg})$ daily for 3-5days.

Subsequently prednisolone (1 $\mathrm{mg} / \mathrm{kg}$ orally) was started and continued for six weeks with gradual tapper. Plasma exchange, intravenous immunoglobulin (IVIG) ( $2 \mathrm{mg} / \mathrm{kg}$ divided dose over 5 days) or repeat high dose intravenous methyl prednisolone were given for patient who continued to detoriate. Data thus computed were analyzed and inferences drawn.

Analysis of data: Results were expressed as mean \pm standard deviation for continuous variables and as number $(\%)$ for categorical data. Since all data were normally distributed, the parametric tests were used for statistical analyses.

The data was analyzed by SPSS version 21 software along with below mentioned appropriate statistical tests at $5 \%$ level of significance. $p$ value is calculated using Chi-Square Test given by the formulaWhere $\mathrm{Oi}$ is Observed frequency and Ei is Expected frequency.

Significance was interpreted as: $0.05<\mathrm{p}<0.10$ Suggestive of significance, $0.01<\mathrm{p}<0.05$ moderately significant, $\mathrm{p}<0.01$ strongly significant.

\section{Observation}

Table-1: Age\& Sex distribution of study subjects.

\begin{tabular}{|c|c|c|c|}
\hline \multirow{2}{*}{ Age in year } & \multicolumn{2}{|c|}{ Sex } & \multirow{2}{*}{ Female } \\
\cline { 2 - 4 } & Male & 5 & 12 \\
\hline 0-4Years & 7 & 7 & 20 \\
\hline 5-9years & 13 & 1 & 05 \\
\hline 10-14years & 5 & $\mathbf{1 3}$ & $\mathbf{3 7}$ \\
\hline
\end{tabular}

Age distribution of study sample has been analyzed. It shows maximum prevalence in 5- 9 year age group (54.05\%) with $32.43 \%$ in $0-4$ year\& $13.52 \%$ in $10-14$ years age group.

Male predominates in the study $64.86 \%$ with female being $35.14 \%$. 
Table-2: Monthly distribution of study subjects.

\begin{tabular}{|c|c|c|}
\hline Month & No. & $\mathbf{\%}$ \\
\hline January & 6 & 16.3 \\
\hline February & 1 & 2.7 \\
\hline March & 2 & 5.4 \\
\hline April & 1 & 2.7 \\
\hline May & 1 & 2.7 \\
\hline June & 2 & 5.4 \\
\hline July & 5 & 13.5 \\
\hline August & 1 & 2.7 \\
\hline September & 4 & 10.8 \\
\hline October & 5 & 13.5 \\
\hline November & 5 & 13.5 \\
\hline December & 4 & 10.8 \\
\hline Total & $\mathbf{3 7}$ & $\mathbf{1 0 0 . 0}$ \\
\hline
\end{tabular}

Maximum cases were seen in the month of January.

Table-3: Presenting Symptoms $(n=37)$

\begin{tabular}{|c|c|c|}
\hline Symptoms & Number & Percentage \\
\hline Convulsion & 24 & 64.9 \\
\hline Fever & 22 & 59.5 \\
\hline Altered sensorium & 21 & 56.8 \\
\hline Paralysis & 16 & 43.2 \\
\hline Vomiting & 9 & 24.3 \\
\hline Headache & 6 & 16.2 \\
\hline Speech abnormality & 6 & 16.2 \\
\hline Bowel \& bladderchanges & 4 & 10.8 \\
\hline Abnormal movement & 3 & 8.1 \\
\hline Blurring of vision & 2 & 5.4 \\
\hline Rash & 2 & 5.4 \\
\hline Double vision & 1 & 2.7 \\
\hline Dysphagia & 1 & 2.7 \\
\hline Neck retraction & 1 & 2.7 \\
\hline Dizziness & 1 & 2.7 \\
\hline
\end{tabular}

The variety of symptoms presented by the study sample at the onset was analyzed here. Seizures were documented to occur in highest no. Ofcases (64.9\%). Next fever (59.5\%) \& altered sensorium $(56.8 \%)$ followed.

Table-4: Pattern of neurological involvement.

\begin{tabular}{|c|c|c|}
\hline Sign & Number & Percentage \\
\hline Motor deficit & 25 & 67.7 \\
\hline Encephalopathy & 32 & 86.5 \\
\hline Autonomic involvement & 15 & 40.5 \\
\hline Cranial nerve involvement & 10 & 27 \\
\hline Cerebellar sign & 8 & 21.6 \\
\hline Aphasia & 6 & 16.2 \\
\hline Meningeal sign & 5 & 13.5 \\
\hline Involuntary movement & 3 & 8.1 \\
\hline
\end{tabular}

Encephalopathy was observed in $86.5 \%$, followed by motor deficit in $67.6 \%$ \& autonomicinvolvementin $40.5 \%$. 
Table-5: Pattern of MRI abnormality.

\begin{tabular}{|c|c|c|}
\hline MRI abnormality & Number & Percentage \\
\hline T1 hypointensity & 17 & 51.52 \\
\hline T2 hypointensity & 25 & 75.76 \\
\hline FLAIR hyperintensity & 24 & 72.73 \\
\hline DWI restriction & 16 & 48.48 \\
\hline Temporal shrinkage & 5 & 6.06 \\
\hline Contrast enhancement & 2 & 6.06 \\
\hline Gyral thickening & 2 & 6.06 \\
\hline Sinusitis & 2 & 3.03 \\
\hline Perifocal edema/mass effect & 1 & 9.09 \\
\hline T2hyperintensity of spinal cord & 3 & 3.03 \\
\hline Normal & 1 & 15 \\
\hline
\end{tabular}

Out of 37\% cases, MRI was done in 33 cases, CT scan was done in 1 case, 3 could not be done. T1 hypointensity was observed in $51.52 \%$, T2 hypointensity in $75.76 \%$,FLAIRS changes in $72.73 \%$ cases.

Table-6: Area of brain involved.

\begin{tabular}{|c|c|c|}
\hline Area of brain & Number & Percentage \\
\hline Fontal lobe & 19 & 55.9 \\
\hline Temporal lobe & 18 & 52.9 \\
\hline Parietal lobe & 17 & 50 \\
\hline Occipital lobe & 13 & 38.2 \\
\hline Thalamus & 6 & 17.6 \\
\hline Basal ganglia & 5 & 14.7 \\
\hline Centrum Semiovale & 5 & 14.7 \\
\hline Cerebellum & 4 & 11.8 \\
\hline Subcortex & 3 & 8.8 \\
\hline Corona radiata & 3 & 8.8 \\
\hline Midbrain & 3 & 8.8 \\
\hline Pons & 2 & 5.9 \\
\hline Occipitoperitrigonal region & 2 & 5.9 \\
\hline U fibre & 1 & 2.9 \\
\hline Medulla & 1 & 2.9 \\
\hline Perisylvian region & 1 & 2.9 \\
\hline Infrasylvian region & 1 & 2.9 \\
\hline Cervical cord & 1 & 2.9 \\
\hline Medullary cord & 1 & 2.9 \\
\hline Entire cord & 1 & 2.9 \\
\hline Meninges & 1 & 2.9 \\
\hline
\end{tabular}

Frontal lobe was involved in maximum number of cases $(55.9 \%)$. 
Table-7: Descriptive Statistic of Cerebrospinal Fluid.

\begin{tabular}{|c|c|c|c|c|c|c|c|c|}
\hline CSF & $\mathbf{N}$ & Class & Number & Percentage & Range & Minimum & Maximum & Stddeviation \\
\hline \multirow{3}{*}{$\begin{array}{c}\text { Cell } \\
\text { Count }\end{array}$} & \multirow{3}{*}{35} & $<5$ & 20 & 57.14 & \multirow{3}{*}{0} & \multirow{3}{*}{100} & \multirow{3}{*}{13.51} & \multirow{3}{*}{20.77} \\
\hline & & $5-20$ & 6 & 14.14 & & & & \\
\hline & & $>20$ & 9 & 25.71 & & & & \\
\hline \multirow{2}{*}{ Protein } & \multirow{2}{*}{35} & $<45$ & 25 & 71.43 & \multirow{2}{*}{15} & \multirow{2}{*}{135} & \multirow{2}{*}{57.86} & \multirow{2}{*}{25.35} \\
\hline & & $>45$ & 10 & 28.57 & & & & \\
\hline \multirow{2}{*}{ Sugar } & \multirow{2}{*}{35} & $<2 / 3(50)$ & 24 & 68.57 & \multirow{2}{*}{0} & \multirow{2}{*}{102} & \multirow{2}{*}{59.43} & \multirow{2}{*}{21.12} \\
\hline & & $>^{2 / 3}(50)$ & 11 & 31.43 & & & & \\
\hline
\end{tabular}

CSF was done in 35 out of 37 cases, pleocytosis was observed in $42.85 \%$ cases, elevated protein in $71.43 \%$ cases \& low sugar in $31.43 \%$ cases.

Table-8: Response to treatment.

\begin{tabular}{|c|c|c|c|c|c|c|c|c|}
\hline \multirow{2}{*}{ Treatment } & \multicolumn{2}{|c|}{ Complete recovery } & Partial /No recovery & \multicolumn{2}{|c|}{ Death } & \multicolumn{2}{c|}{ Total } \\
\cline { 2 - 9 } & No & $\%$ & No & $\%$ & No & $\%$ & No & $\%$ \\
\hline Steroid & 9 & 3 & 18 & 62 & 2 & 7 & 29 & 100 \\
\hline Steroid +IVIG & - & - & 1 & 50 & 1 & 50 & 2 & 100 \\
\hline $\begin{array}{c}\text { Only Supportive } \\
\text { treatment }\end{array}$ & 31 & 50 & - & - & 3 & 50 & 6 & 100 \\
\hline
\end{tabular}

Steroid were given in only 29 number of patient, steroid plus IVIG were given in 2 patient, only supportive treatment were given in 6 out of 37 patients.

Table-9: Pattern of sequelae on follow up $(n=17)$.

\begin{tabular}{|c|c|c|}
\hline Sequelae & Number & Percentage \\
\hline Seizure & 9 & 52.9 \\
\hline Motor deficit & 8 & 47 \\
\hline Cognitive dysfunction & 4 & 23.5 \\
\hline Behavioural disorder & 4 & 23.5 \\
\hline Dysphasia & 3 & 17.6 \\
\hline Dysphasia & 1 & 5.9 \\
\hline
\end{tabular}

Out of 17 children with sequelae, epilepsy was predominantly seen $52.9 \%$ of cases.Motor deficit is seen in $47 \%$ of cases.

Table-10: Association of predisposing factor with respect to age, severity of presentation, outcome.

\begin{tabular}{|c|c|c|c|c|c|c|c|c|c|c|c|c|c|c|c|c|}
\hline \multirow{3}{*}{$\begin{array}{c}\text { Age } \\
\text { gp } \\
\text { years }\end{array}$} & \multicolumn{4}{|c|}{ Predisposing factor } & \multicolumn{6}{|c|}{ Severity of presentation } & \multicolumn{6}{|c|}{ Outcome } \\
\hline & \multicolumn{2}{|c|}{ Present } & \multicolumn{2}{|c|}{ Absent } & \multicolumn{2}{|c|}{ Mild } & \multicolumn{2}{|c|}{ Moderate } & \multicolumn{2}{|c|}{ Severe } & \multicolumn{2}{|c|}{ Complete } & \multicolumn{2}{|c|}{ Partial } & \multicolumn{2}{|c|}{ Death } \\
\hline & No & $\%$ & No & $\%$ & No & $\%$ & No & $\%$ & No & $\%$ & No & $\%$ & No & $\%$ & No & $\%$ \\
\hline $0-4$ & 9 & 81.8 & 2 & 18.2 & 2 & 16.66 & 5 & 41.67 & 5 & 41.67 & 3 & 25 & 8 & 40 & 1 & 20 \\
\hline $5-9$ & 8 & 47.1 & 9 & 52.9 & 6 & 30 & 6 & 30 & 8 & 40 & 7 & 58.33 & 9 & 45 & 4 & 80 \\
\hline $10-14$ & 4 & 80 & 1 & 20 & 1 & 20 & 3 & 60 & 1 & 20 & 2 & 16.67 & 3 & 15 & 0 & 0 \\
\hline Total & 21 & 63.6 & 12 & 27.4 & 9 & 24.32 & 14 & 37.84 & 14 & 37.84 & 12 & 100 & 20 & 100 & 5 & 100 \\
\hline
\end{tabular}

Chi square $=4.947, \mathrm{p}=048 \mathrm{in}$ predisposing factor, chi square $=1.612, \mathrm{p}=0.807$ inseverity of presentation, chi square $=2.372$, $\mathrm{p}=0.668$ outcome at discharge. 
*Predisposing factors [Respiratory infection (38\%), Nonspecific fever (19.1\%), GI infection (9.5\%),UTI (9.5\%), CNS infection (9.5\%), Measles (4.8\%), Mumps (4.8\%), appendicitis (4.8\%)].

Although the prevalence of predisposing factor seems to be higher in 0-4years age group, it is not confirmed statistically. It seems that 0-4 years had mostly moderate to severe presentation and 5-9 year age group had mostly severe presentation. Statistically significant association could not be established. There is no statistically significance as seen with outcome at discharge.

Table-11: Association of severity of presentation with outcome at discharge.

\begin{tabular}{|l|c|c|c|c|c|c|c|c|}
\hline \multirow{2}{*}{ Outcome at discharge } & \multicolumn{9}{|c|}{ Glassgow coma score } & \multicolumn{2}{c|}{ Total } \\
\cline { 2 - 10 } & \multicolumn{2}{|c|}{ Mild } & \multicolumn{2}{c|}{ Moderate } & \multicolumn{2}{c|}{ Severe } & \multicolumn{2}{c|}{} \\
\cline { 2 - 10 } & No & $\mathbf{\%}$ & No & $\mathbf{\%}$ & No & $\mathbf{\%}$ & No & $\%$ \\
\hline Complete recovery & 5 & 55.56 & 4 & 28.57 & 3 & 21.43 & 12 & 32.43 \\
\hline Partial or no recovery & 4 & 44.44 & 10 & 71.43 & 6 & 42.86 & 20 & 54.05 \\
\hline Death & 0 & 0 & 0 & 0 & 5 & 35.71 & 5 & 13.51 \\
\hline Total & $\mathbf{9}$ & $\mathbf{1 0 0}$ & $\mathbf{1 4}$ & $\mathbf{1 0 0}$ & $\mathbf{1 4}$ & $\mathbf{1 0 0}$ & $\mathbf{3 7}$ & $\mathbf{1 0 0}$ \\
\hline
\end{tabular}

Chi square $=11.54, \mathrm{p}=0.021$

It was observed that the cases with severe presentation had lower proportion of recovery (21.43\%) \& higher proportion of mortality $(35.71 \%)$ which was found to be statistically significant.

\section{Discussion}

37 children were included into the study who have evidence of CNS demylination; 36 fulfilled the criteria for first episode of ADEM \& 1 was diagnosed as multiple sclerosis. Out of 10701 indoor admissions during period ADEM constituted $0.33 \%$ of admitted cases.

ADEM was found in all age group of children with early childhood predominance. The youngest was 45 days old infant and oldest was 14 years old. The peak incidence was observed in 5-9 years age group with mean age presentation being 5.2 years \pm 3.44 . Studies in the past have reported comparable findings $[3,5,8]$.

Boys constituted $65 \%$ of the children in the present study and girls constituted 35\%. Studies from India and abroad have reported that ADEM is more common in boys, while multiple sclerosis is more common in girls $[4,5]$. Cases occurred throughout the year with predominance in the month of January which were comparable to the western data where predominance during winter and spring is reported[3,4].

The clinical feature of ADEM in the present sample were comparable to those of previous reports. Seizures $(65 \%)$ followed by fever $(60 \%)$ and altered sensorium $(57 \%)$ were most common presenting symptoms $[3,4,9,10]$. Encephalopathy (87\%) and motor deficit $(68 \%)$ were most frequent neurological finding.
MRI is of central importance in the diagnosis of acute CNS white matter disorder. CT is frequently normal in ADEM (Dunn et al 1986 [11], caldemeyer et al 1994 [12]). About 05 patient in our study had CT scan done that showed features suggestive of demyelination in 4 patients.

Abnormalities of brain MRI have been shown to be predictive of the development of multiple sclerosis in adults. In clinically isolated syndromes an abnormal MRI brainon presentation was associated with progression of multiple sclerosis in $83 \%$ of patients during last10years of follow up (O'Riordan et al 1998)[13].

Normal MRI was associated with progression to multiple sclerosis in our only patient during the study period. 33 out of 37 children in this study group had MRI done which was abnormal in presentation in all except one. T1 hypointensity was noted in $52 \%$ cases and T2 \& FLAIR hyper intensity was noted in $76 \%$ cases.

Temporal shrinkage was noted in 05 patient (15\%), contrast enhancement in $2(6 \%)$ \& gyral thickening in $2(6 \%)$ Lobes of the brain were the most common area to be involved out of which fontal is the most frequent $(56 \%)$. T2 hyperintensity of spinal cord was noted in three of our patients who had a screening MRI spine 
done, entire cord is involved in one, cervical in one and medulla in one. Review of multiple sclerosis in the children in past have documented the predominance of periventricular lesions (near universal), particularly at the trigone and the body of lateral ventricle (Alper G et al 2009)[14]. Cortical grey lesions were infrequent.

Involvement of deep grey matter was also seen occurring in $32 \%$ cases of our study which may be the only imaging finding in ADEM (Baum et al 1994[15], Kimura et al 1996[16]). The asymmetry found in the white matter lesions are characteristics of acquired demyelinating lesion. Symmetrical white matter abnormalities should prompt consideration of leucody strophy. In contrast the deep grey matter abnormalities in ADEM are frequently symmetrical. Thalamic involvement was seen in $18 \%$ of cases, which can be up to $40 \%$ as per previous studies $[3,4,12,17]$.

Evidence of CSF inflammations in the form of pleocytosis $(43 \%)$ and elevated protein $(71 \%)$ was seen which was described in $28-65 \%$ of studied cases of ADEM $[3,4,18]$. CSF opening pressure was normal in almost all cases baring a few. Cells were moderately elevated with mean cell countbeing 14/cumm ranging from 0-100/ cumm which were predominantly mononuclear.

Current opinion supports the use of intravenous methyl prednisolone followed by oral prednisolone therapy when infective encephalitis has been excluded and acute post infectious demyelination is suspected. Notably, however some children appear to recover fully without any therapeutic intervention, but recovery is usually incomplete $[19,20]$. No therapy (only supportive) was given in 6 patients in our study, some due to fulminant presentation that hardly give any time for intervention. Out of 31 patients who were treated only steroid was given in 29 of them and combined therapy of steroid \&IVIG was given in 2.Therapeutic intervention was random, but previous studies have shown efficacy of various therapeutic modalities in specific area of involvement [21].

On follow up, complete recovery was seen in 11 subjects $(38 \%)$; sequelae were found in 17 subjects $(59 \%) ; 1$ succumbed. 3 patients were lost to follow up. Out of 12 patient who had recovered completely at the time of discharge, 2 had sequelae in the form of behavioural problem and need for anti-epileptic drugs. Out of 20 patients who had partial or no recovery at the time of discharge ,1 succumbed,2 had complete recovery, residual neurological deficit were seen in rest others in the form of cognitive dysfunction, recurrent seizures, motor deficit, behavioural problem, dysphasia $\&$ dysphagia. Predominant sequelae observed were recurrent seizures $(53 \%)$ \& moor deficit (47\%). In patient who had complete recovery, the recovery period ranged from 8days to maximum 3 months, recovery may continue from weeks to months as per previous studies [22].

The preceding illness were described as upper respiratory tract infection (8), nonspecific fever (4), GI infection (2), UTI (2), CNS infection (2), measles (1), mumps (1) and appendicitis (1), the majority of patient had a preceding history of upper respiratory $\operatorname{tract}(38 \%)$ as per previous studies $[23,24]$. The prevalenceof antecedent illness was mostly observed below 10 years of age $(81 \%)$ with predominance of $0-4$ years age group, the association could not be held statistically significant $(p=0.084)$, this study needs with larger sample to comment on a definite association. In previous studies,the lack of prodrome has been observed $>30 \%$ of adolescent cases [12]. The prevalence of preceding illness is usually less described in multiple sclerosis (R C Dale et al 2000)[4]. The association of age with severity of presentation do not have statistically significant value, need a larger sample. Also the outcome at discharge do not have any statistical significance.

The cases with severity of presentation had lower proportion of complete recovery $(21.43 \%)$ \& higher proportion of mortality $(35.71 \%)$ than the cases with mild and moderate presentation. Similarly, the cases with moderate degree of presentation had lower rate of complete recovery $(28.57 \%)$ than those with mild presentation $(55.56 \%)$, this indicates that the severity of presentation was inversely proportional to recovery at discharge. It was proved to be statistically significant through chi-square test of association with $\mathrm{p}$ value $=0.021$.

\section{Conclusion}

ADEM most commonly present as a polysymptomatic encephalopathy and initially diagnosis may not be clear. Clinical evaluation, MRI \& CSF studies are most useful to establish the diagnosis and rule out important differential diagnosis. There is presence of antecedent illness in case of severe presentation. The age, gender, predisposing factor do not influence the outcome, but the severity of presentation influence the outcome at discharge. Early institution of therapy with immunosuppressive drugs hasten recovery and reduces morbidity as evidence by the study done by Rust RS et al [19], Francis GS et al [20], Ravaglia S [21]. 
The two largest reviews found that disabilities was mild (defined as extended disability score of $<4$ ) in 40 and $60 \%$ of patients in 8 to 10 years of follow up respectively (Dequet et al 1987[25], Ghezee et al 1997 [26]) Children with ADEM need long term follow up for cognitive impairment and emotional problems as described by Boutin et al 1988 [27].

More than half of ADEM case had preceding illness including some vaccine preventable disease which could be prevented by proper vaccination.

What this study adds to existing knowledge?: Inadvertent short course use of steroid therapy is responsible for relapse and subsequent appearance of new symptoms. This study high lights the importance that malaria should be added to the list of infections able to precipitate ADEM. However, large prospective studies are required to address the aetio-pathogenesis.

Funding: Nil, Conflict of interest: None initiated, Perission from IRB: Yes

\section{References}

1. Panicker J N. Acute disseminated encephalomyelitis. Ann Indian AcadNeurol2007;10:137-44

2. Jun-liang Yuan, Shuang-kun Wang, Xiao-juan Guo, and Wen-li Hu.Acute Disseminated Encephalomyelitis following Vaccination against Hepatitis B in a Child: A Case Report and Literature Review.Case Rep Neurol Med. 2016; 2016: 2401809.

3. Hynson JL, Kornberg AJ, Coleman LT, et al. Clinical and neuroradiologic features of acute disseminated encephalomyelitis in children. Neurology. 2001 May 22;56(10):1308-12. DOI:10.1212/wnl.56.10.1308

4. R. C. Dale C. de Sousa W. K. Chong T. C. S. Cox B. Harding B. G. R. Neville. Acute disseminated encephalomyelitis, multiphasic disseminated encephalomyelitis and multiple sclerosis in children. Brain, 2000;123:2407-2422

5. Tenembaum S, Chamoles N, Fejerman N. Acute disseminated encephalomyelitis: a long-term follow-up study of 84 pediatric patients. Neurology. 2002 Oct 22;59(8):1224-31. DOI:10.1212/wnl.59.8.1224

6. Alvord BC jr Demyelinating diseases in Vinken PJ, Bruyen GW(Eds) handbook of clinical neurology Amsterdam; Elsevier publisher BV,1985; 3 (47); 467-502
7. Sonia Madan, S. Aneja, R. P. Tripathi, A. Batra, Anju Seth and Veena Taluja. Acute Disseminated Encephalomyelitis - A Case Series Indian Pediatrics 2005; 42:367-371

8. Anlar B, Basaran C, Kose G, Guven A, Haspolat S, Yakut A, et al. Acute disseminated Encephalomyelitis in Children: Outcome and Prognosis. Neuropediatrics. 2003; 34:194-9

9. Johnson RT, Griffin DE, Hirsch RL, et al. Measles encephalomyelitis--clinical and immunologic studies. N Engl J Med. 1984 Jan 19;310(3):137-41. DOI:10.1056/ NEJM198401193100301

10. Leake JA, Albani S, Kao AS, et al. Acute disseminated encephalomyelitis in childhood: epidemiologic, clinical and laboratory features. Pediatr Infect Dis J. 2004 Aug;23(8):756-64.

11. Dun V, Bale JF Jr, Zimmerman RA, et al. MRI in children with postinfectious disseminated encephalomyelitis. Magn Reson Imaging. 1986; 4 (1): 25-32.

12. Caldemeyer, K.S., Smith, R.R., Harris, T.M. et al. Neuroradiology (1994) 36: 216. https://doi.org/10.1007/ BF00588134

13. O'Riordan JI, Gomez-Anson B, Moseley IF, et al. Long term MRI follow-up of patients with post infectious encephalomyelitis: evidence for a monophasic disease. J Neurol Sci. 1999 Aug 15;167(2): 132-6.

14. Alper G. Acute disseminated encephalomyelitis. J Child Neurol. 2012 Nov;27(11):1408-25. doi: 10.1177/ 0883073812455104. Epub 2012 Aug 21.

15. Baum PA, Barkovich AJ, Koch TK, Berg BO. Deep gray matter involvement in children with acute disseminated encephalomyelitis. Am J Neuroradiol. 1994;15:1275-83.

16. Kimura S, Nezu A, Ohtsuki N, et al. Serial magnetic resonance imaging in children with postinfectious encephalitis. Brain Dev. 1996 Nov-Dec; 18 (6): 461-5.

17. Schwarz S, Mohr A, Knauth M, et al. Acute disseminated encephalomyelitis: a follow-up study of 40 adult patients. Neurology. 2001 May 22;56(10): 1313-8. DOI:10.1212/wnl.56.10.1313 
18. Shahar E, Andraus J, Savitzki D, et al. Outcome of severe encephalomyelitis in children: effect of highdose methylprednisolone and immunoglobulins. J Child Neurol. 2002 Nov;17(11):810-4.

19. Rust RS, Dodson W, Prensky A. Classification and outcome of acute disseminated encephalomyelitis. Ann Neurol. 1997;42:491.

20. Francis GS, Deguette P, Antel JP. Inflammatory demyelinating disease of the central nervous system. In: Bradely WG, Daroff RB, Fenichel GM, editors. Neurol Clin Prac. 3nd ed. Vol. 2. Boston: Butter worth Heinemann; 1995. pp. 1307-1343.

21. Ravaglia S, Piccolo G, Ceroni M, et al. Severe steroid-resistant post-infectious encephalomyelitis: general features and effects of IVIg. J Neurol. 2007 Nov; 254(11):1518-23. Epub 2007 Nov 14.

22. Marchioni E, Marinou-Aktipi K, Uggetti C, et al. Effectiveness of intravenous immunoglobulin treatment in adult patients with steroid-resistant monophasic or recurrent acute disseminated encephalomyelitis. J Neurol. 2002 Jan;249(1):100-4.
23. Menge $\mathrm{T}$, Hemmer $\mathrm{B}$, Nessler $\mathrm{S}$, et al. Acute disseminated encephalomyelitis: an update. Arch Neurol. 2005 Nov;62(11):1673-80. DOI:10.1001/ archneur. 62.11.1673

24. Rust RS. Multiple sclerosis, acute disseminated encephalomyelitis, and related conditions. SeminPediatr Neurol. 2000 Jun;7(2):66-90.

25. Duquette P, Murray TJ, Pleines J, Ebers GC, Sadovnick D, Weldon P, et al. Multiple sclerosis in childhood: clinical profile in 125 patients. J Pediatr. 1987;111:359-363.

26.Ghezzi A, Deplano V, Faroni J, Grasso MG, Liguori M, Marrosu G, et al. Multiple sclerosis in childhood: clinical features of 149 cases. MultScler 1997; 3: $43-6$.

27.Boutin B, Esquivel E, Mayer M, Chaumet S, Ponsot G, Arthuis M. Multiple sclerosis in children: report of clinical and paraclinical features of 19 cases. Neuropediatrics 1988; 19: 118-23.

\section{How to cite this article?}

Jena P.K, Murmu M.C, Priyadarshini D. A study on acute disseminated encephalomyelitis in children. Int J Pediatr Res. 2019;6(05):207-216.doi:10.17511/ijpr.2019.i05.03 